\title{
El caso de la línea 4 del metrobús y la gestión de redes colaborativas urbanas
}

\section{The case of the line 4 of metrobus and managment of collaborative urban networks}

\author{
Edgar E. Ramírez-de la Cruz* \\ Manlio F. Castillo-Salas**
}

\begin{abstract}
This paper addresses the effectiveness of urban governance networks. The case study is the management process of the Line 4 of Metrobus, in Mexico City's downtown. We found that when network structure is not appropriate in terms of its effectiveness, given the context of public policy, managers may use their skills to successfully steer the network to its purposes. It was also found that consensus on which instruments should be used by the network may be as important to manage it properly as the agreement about its global goals. In this context, required network-level competencies were shown to be higher than expected by theory.
\end{abstract}

Keywords: urban management, governance networks, networks management.

\section{Resumen}

Este artículo analiza la efectividad en la gestión de redes de gobernanza urbana, utilizando como laboratorio el caso de la Línea 4 del Metrobús de la Ciudad de México. Encontramos que cuando la configuración de la red no es la más adecuada en función de su efectividad, los gestores utilizan sus capacidades para dirigir la red hacia sus propósitos. También se advierte que el consenso sobre los instrumentos utilizables por la red puede ser tan relevante para gestionarla adecuadamente como el acuerdo acerca de sus objetivos globales. En este entorno, las competencias requeridas a nivel de red son mayores a lo previsto por la teoría.

Palabras clave: gestión urbana, redes de gobernanza, gestión de redes.

* Centro de Investigación y Docencias Económicas, División de Administración Pública, México. Correo-e: edgar.ramirez@cide.edu

** Centro de Investigación y Docencias Económicas, División de Administración Pública, México. Correo-e: mancasals@gmail.com 


\section{Introducción}

Las grandes zonas urbanas o áreas metropolitanas se caracterizan por presentar dos tipos de fragmentación: una de ellas es territorial y se produce por la separación ficticia que imponen las fronteras político-administrativas de las unidades sub-metropolitanas, las cuales no se adaptan fácilmente al crecimiento físico de la urbe (Ramírez, 2012). Por otro lado, la complejidad social existente en las ciudades provoca una fragmentación causada por la heterogeneidad de intereses que conviven en ellas, pues es frecuente que diversos agentes defiendan sus propias preferencias e ideas acerca de los problemas públicos urbanos, tratando de intervenir en su definición y solución. Así, la heterogeneidad fracciona los esfuerzos sociales para llevar a la ciudad sobre una ruta determinada. Este segundo tipo de fragmentación es el centro de interés del presente estudio.

Esta investigación explora en qué medida la gestión pública, por medio de redes colaborativas, constituye una alternativa para la solución eficaz de problemas públicos, en particular, cuando busca remediar la fragmentación creada en ámbitos políticos donde participan grupos con un alto grado de heterogeneidad. Para este estudio es primordial preguntarse y analizar cómo los mecanismos de colaboración para el gobierno urbano alcanzan los propósitos que se plantean. Este tema se explora aquí desde las perspectivas de la gobernanza urbana y de la gestión pública a través de redes, identificando el papel que asumen los actores que buscan conducir estas estructuras colaborativas, las cuales, a su vez, pueden ofrecer diferentes retos a los gestores debido a su configuración y a su interacción con el contexto.

La investigación se basa en el análisis de la gestión que realizó la Autoridad del Centro Histórico de la Ciudad de México (ACH) en la implementación de la Línea 4 del Metrobús ${ }^{1}$ entre 2011 y 2012. En la culminación exitosa de este proyecto, la $\mathrm{ACH}$ desempeñó un papel fundamental al gestionar recursos y relaciones de colaboración con otras agencias del gobierno de la Ciudad de México, así como con organizaciones privadas y ciudadanas.

Con el fin de presentar los resultados de la investigación y el marco conceptual, el documento se organiza de la siguiente manera. En primer lugar, se presentan brevemente algunas de las contribuciones del análisis de redes al estudio de la gestión y las políticas públicas. En la segunda sección se plantea el caso de estudio. A continuación, se exponen los conceptos sobre gestión de redes que serán empleados en el análisis, así

\footnotetext{
${ }^{1}$ Metrobús es la denominación que recibe el sistema Bus Rapid Transit (BRT) en el Distrito Federal. Consiste en autobuses articulados que circulan por rutas y carriles exclusivos. Para mayor información acerca de esta modalidad de transporte se recomienda consultar a Wright et al. (2010).
} 
como las proposiciones que orientan el examen de la evidencia. La parte cuatro describe la metodología y los resultados observados. En la quinta sección se discuten los hallazgos y, por último, se presentan algunas conclusiones preliminares sobre las prácticas de gestión de redes.

\section{La gestión de redes colaborativas}

El concepto de redes es de gran utilidad para estudiar fenómenos en los que la estructura de relaciones entre las unidades analizadas (individuos, organizaciones, agencias gubernamentales, etcétera) tiene tanto o mayor efecto que los atributos o características de dichas unidades para explicar un determinado fenómeno (Ramírez, 2016).2 En la década de los 90, y con más intensidad desde los últimos diez años, el uso y la importancia de este concepto, así como su operacionalización mediante el Análisis de Redes Sociales han ido en aumento en el estudio de la administración y la gestión públicas (Berry et al., 2004; O’Toole, 1997; Provan y Kenis, 2008; Provan y Milward, 1995; Ramírez, 2016; Salancik, 1995).

El concepto de redes facilita examinar las diversas estructuras relacionales y su potencial contribución a la cooperación entre organizaciones públicas, no lucrativas y privadas, así como estudiar la capacidad de los gerentes públicos para gestionar tales estructuras (Agranoff, 1991, 2003; O’Toole, 1997). Por otra parte, la gestión de redes también está estrechamente ligada con el proceso de gobernanza. La relación ocurre en la medida en que ambos conceptos se refieren al proceso de conducirlas u organizarlas. Por ejemplo, el concepto de gobernanza de redes ha sido utilizado descriptivamente para ilustrar la interdependencia y las diversas relaciones que existen entre organizaciones públicas, privadas y civiles (Rhodes, 2000).

Para definir un adecuado modelo de gestión de las redes colaborativas y las funciones del gerente, se deben considerar las características del entorno de la red, Por lo tanto, una discusión acerca de la efectividad de las redes debe abarcar no solamente el papel de quienes administran y conducen la red, sino también las contingencias que llevan al establecimiento de determinadas formas de gobernanza. Con el fin de contribuir al debate sobre la efectividad de las redes y de ciertas formas de gobernanza que aquéllas adquieren, proponemos examinar si las funciones de gestión de una red identificadas por McGuire (2002)" corresponden con las prácticas observadas en el caso de la $\mathrm{ACH}$. En particular, se busca reconocer si esas prácticas se ajustan con las necesidades de una de las

\footnotetext{
${ }^{2}$ O’Toole (1997: 45) definió las redes como "estructuras de interdependencia que involucran varias organizaciones o partes de ella, donde ninguna unidad está subordinada a otra en algún acuerdo jerárquico más amplio".
} 
estructuras de gobernanza de red, es decir, de una red gestionada por una organización líder (Provan y Kenis, 2008).

\section{La Autoridad del Centro Histórico y la construcción de la Línea 4 del Metrobús}

El Centro Histórico de la Ciudad de México fue delimitado territorialmente por las autoridades en 1980, y declarado Patrimonio de la Humanidad en 1987 por la Organización de las Naciones Unidas para la Educación, la Ciencia y la Cultura (UNESCO). Desde entonces, cualquier proyecto que modifique de manera importante esta área de la ciudad debe mantener un balance entre la conservación y la funcionalidad. Por esta razón, el principal reto de la autoridad ha sido preservar los edificios antiguos y las áreas públicas en su esplendor original y, al mismo tiempo, permitir que los espacios puedan ser habitados y utilizados para actividades comerciales o de servicios.

Con el fin de facilitar los procesos de gestión en el Centro Histórico, el gobierno de la ciudad instituyó, en 2007, un órgano de apoyo adscrito a la Jefatura de Gobierno del Distrito Federal, denominado Autoridad del Centro Histórico (ACH). Según su Acuerdo de Creación, ${ }^{3}$ la $\mathrm{ACH}$ tiene diversas atribuciones en materia de gobierno, desarrollo urbano y vivienda, desarrollo económico, medio ambiente, obras y servicios, desarrollo social, transportes y vialidad, turismo, cultura y seguridad pública; aunque sus principales encargos pueden resumirse en dos: coordinar las iniciativas que conciernen al territorio del Centro Histórico, asumiendo el mando operativo de las unidades administrativas del Gobierno del Distrito Federal (GDF) establecidas en ese sitio; ${ }^{4}$ y diseñar políticas específicas para el desarrollo de esta zona de la ciudad.

En el marco de sus atribuciones, la $\mathrm{ACH}$ tuvo un papel central en la convocatoria y la coordinación de los distintos actores involucrados en el proyecto de la Línea 4 del Metrobús, a través de una mesa de discusión expresamente creada para ese propósito. Además de la propia $\mathrm{ACH}$, en la mesa participaron otras dependencias del GDF como la Secretaría de Transporte y Vialidad (Setravi), la Secretaría de Obras Públicas y Servicios (SOPS), la Secretaría de Seguridad Pública (SSP) y la Secretaría de Gobierno.

En la Ciudad de México, la adopción del Metrobús ha implicado modificaciones en el entorno urbano, en las costumbres de los usuarios del transporte colectivo y en las formas de organización de los concesio-

\footnotetext{
${ }^{3}$ Publicado en la Gaceta Oficial del Distrito Federal, el 22 de enero de 2007.

${ }^{4}$ Artículo Cuarto del Acuerdo de Creación de la Autoridad del Centro Histórico.
} 
narios del sector. Después de ocho años de funcionamiento y de ofrecer un servicio que está bien evaluado por los usuarios, el escepticismo inicial del público y de los empresarios del transporte acerca del Metrobús ha disminuido. Sin embargo, no puede decirse que las dudas sobre su funcionamiento, eficiencia y rentabilidad hayan terminado por completo, por lo que el anuncio de una nueva línea para la ampliación del servicio genera reacciones entre los distintos grupos que se sienten beneficiados o perjudicados por una obra como ésta.

Desde 2005, el GDF ha puesto en marcha seis corredores de Metrobús en la Ciudad de México. La Línea 4 pasa por el Centro Histórico de la ciudad y su establecimiento fue un proceso particularmente complejo, no sólo por la pluralidad de actores interesados en el proyecto, sino por transitar en medio de una zona considerada como Patrimonio Cultural de la Humanidad.

Luego de desechar la construcción de tranvías, debido a su elevado costo, el GDF decidió que la modernización de la movilidad en el Centro Histórico sería a través del sistema Metrobús, aunque con algunas adaptaciones en el diseño de las rutas y las estaciones, con el fin de alterar lo menos posible la imagen urbana del área. La Secretaría de Obras Públicas y Servicios del GDF anunció que, además de las ventajas en función de la movilidad, la Línea 4 mejoraría el entorno aledaño al corredor al reforzar el alumbrado y el mobiliario urbano, rehabilitar las jardineras y mejorar los señalamientos para los peatones. La construcción de esta línea se enmarcó en los objetivos del Programa Integral de Transporte y Vialidad 2007-2012.

Después del anuncio del proyecto, las principales reacciones provinieron de tres sectores interesados: los grupos de comerciantes (ambulantes y establecidos) del Centro Histórico, los transportistas o concesionarios que operaban las rutas de autobús que transitaban por el centro y algunos actores políticos que forman parte de los partidos de oposición al Gobierno de la Ciudad. El hecho es remarcable al menos por dos razones. La primera es que puso en evidencia a una pluralidad de grupos de interés que existen en el Centro Histórico de la Ciudad de México, y que poseen una importante capacidad para oponerse a una decisión gubernamental. Y, en segundo lugar, porque el Gobierno de la Ciudad reconoció que no podía imponer su punto de vista fácilmente, sino que necesitaba de una importante labor de gestión para satisfacer los reclamos que consideraba legítimos, y para anular la oposición política al proyecto. 


\section{Proposiciones}

La efectividad de una red de gobernanza como la del Centro Histórico de la Ciudad de México depende, en buena medida, de las habilidades prácticas y el conocimiento con los que se gestione. ${ }^{5} \mathrm{Al}$ respecto, McGuire (2002) ofrece una serie de hipótesis sobre el comportamiento y las prácticas de los gerentes de redes, señalando que pueden estimular ciertas conductas de colaboración para llevar a la red hacia el cumplimiento de sus objetivos. McGuire (2002 y 2006) explica que los tipos de actividades que se deben realizar en la gestión colaborativa son al menos cuatro: activación, encuadramiento, movilización y sintesis. La activación es la identificación de los actores y de los recursos de todo tipo, necesarios para alcanzar los propósitos del arreglo. El encuadramiento se refiere al acuerdo de los actores sobre el funcionamiento y las reglas de la red. La movilización es la motivación y definición de compromisos reales entre los actores para llevar a cabo conjuntamente las tareas de la red. Finalmente, la sintesis se refiere a una interacción virtuosa y productiva entre todos los actores, que tiende a reforzar la confianza y el flujo de información entre ellos (McGuire, 2006).

Sin embargo, es necesario destacar que la actuación de los gerentes no es el único factor que influye en el desempeño de la red. Provan y Kenis (2008) argumentan que diferentes combinaciones de factores como la confianza, el número de participantes, el grado de consenso en los objetivos y las capacidades o competencias ${ }^{6}$ de la red permiten que diferentes tipos de estructuras de gobernanza de redes sean más o menos efectivas. Los mismos autores clasifican tres formas de gobernanza de redes, de acuerdo con las particularidades de cada tipo de estructura. La gobernan$z a$ compartida se caracteriza por el autogobierno, en el que participan todos los integrantes de la red. La gobernanza mediante una organización líder funciona mejor en redes intermediadas, donde el mediador es una organización que, perteneciendo a la red, tiene capacidad y recursos para ejercer el liderazgo de la misma. En estas redes, el consenso sobre los objetivos tiende a ser bajo, porque la organización líder procura alinearlos con los suyos. Por último, la gobernanza mediante una organización administrativa también parece funcionar mejor en redes intermediadas, con la diferencia de que el mediador o gestor de la red es externo a ella. Los predictores esenciales de cada tipo de red se presentan en la tabla 1.

\footnotetext{
${ }^{5}$ Debe notarse que nos referimos a una red creada deliberadamente para cumplir ciertos objetivos.

${ }^{6}$ Es decir, las habilidades necesarias para que la red realice sus labores y, en general, para que funcione correctamente.
} 


\section{Tabla 1}

\section{Predictores clave de la efectividad de las formas de gobernanza de redes}

\begin{tabular}{lllll}
\hline \multicolumn{1}{c}{$\begin{array}{c}\text { Forma de } \\
\text { gobernanza }\end{array}$} & \multicolumn{1}{c}{ Confianza } & $\begin{array}{c}\text { Número de } \\
\text { participantes }\end{array}$ & $\begin{array}{c}\text { Consenso sobre el } \\
\text { objetivo }\end{array}$ & $\begin{array}{l}\text { Necesidad de } \\
\text { competencias } \\
\text { a nivel de red }\end{array}$ \\
\hline $\begin{array}{l}\text { Gobernanza } \\
\text { compartida }\end{array}$ & $\begin{array}{l}\text { De alta } \\
\text { densidad }\end{array}$ & Pocos & Alto & Baja \\
$\begin{array}{l}\text { Mediante una } \\
\text { organización }\end{array}$ & $\begin{array}{l}\text { De baja densidad, } \\
\text { altamente } \\
\text { líder }\end{array}$ & Moderado & $\begin{array}{l}\text { Moderadamente } \\
\text { centralizada }\end{array}$ & Moderada \\
$\begin{array}{l}\text { Mediante una } \\
\text { organización } \\
\text { administrativa }\end{array}$ & $\begin{array}{l}\text { De moderada } \\
\text { densidad, la orga- } \\
\text { nización adminis- } \\
\text { trativa es moni- } \\
\text { toreada por los } \\
\text { miembros de la red }\end{array}$ & De moderado & $\begin{array}{l}\text { Moderadamente } \\
\text { alto }\end{array}$ & Alta \\
& & & \\
\hline
\end{tabular}

Fuente: tomado de Provan y Kenis (2008: 237).

Dado el mandato y las funciones que se le asignaron a la $\mathrm{ACH}$, se puede sostener -como una primera proposición a explorar- que ésta se diseñó para funcionar como una organización líder en la red de actores públicos y privados que interactúan en el Centro Histórico de la Ciudad de México. Con este antecedente, la primera parte de la investigación está dedicada a examinar las características de la red, con el fin de contrastar si sus características en términos de confianza, participantes, consenso sobre objetivos y necesidades de competencias se asemejan al modelo teórico, lo que proporciona elementos para discutir la pertinencia de su diseño en correspondencia con las características presentadas en la tabla 1.

Por otra parte, partiendo del supuesto de que la $\mathrm{ACH}$ buscaba lograr un desempeńo eficaz de la red (es decir, la implementación de la Línea 4), para el caso que nos ocupa deberíamos esperar que se verificaran las siguientes proposiciones, las cuales se relacionan con las prácticas o actividades de gestión que realizó la $\mathrm{ACH}$, y están basadas en las hipótesis de McGuire (2002 y 2006):

1. Las características de la red del Centro Histórico eran compatibles con los rasgos de la "gobernanza mediante una organización líder" que impulsó el GDF a través de la $\mathrm{ACH}$, en términos de la confianza entre los participantes, su número, el consenso sobre los objetivos y la necesidad de competencias a nivel de red. 
2. Si la red del Centro Histórico tenía objetivos claros, la ACH debió enfocar sus recursos de gestión hacia la activación y la síntesis para conseguir la implementación de la Línea 4.

3. Si la red del Centro Histórico tenía objetivos poco claros o muy variados, la $\mathrm{ACH}$ debió dirigir sus recursos de gestión hacia la movilización y el encuadramiento para conseguir la implementación de la Línea 4.

4. Si los miembros de la red del Centro Histórico habían trabajado juntos en proyectos anteriores, la $\mathrm{ACH}$ debió dirigir menos recursos de gestión a las actividades de síntesis.

5. Si la red del Centro Histórico tenía objetivos relativamente claros, la $\mathrm{ACH}$ debió enfocar gran parte de sus recursos de gestión a explotar el ambiente externo a favor de la Línea 4, más que a estabilizar el sistema al interior de la red.

6. Si los actores clave para llevar a cabo el proyecto de la Línea 4 mostraban poco apoyo al mismo, la $\mathrm{ACH}$ debió enfocar sus recursos de gestión en la movilización.

7. Si los recursos para llevar a cabo la implementación de la Línea 4 se encontraban dispersos, la $\mathrm{ACH}$ debió dirigir sus recursos de gestión hacia la activación y la movilización.

8. Si la ejecución del proyecto de la Línea 4 dependía de la disposición de recursos locales o de los miembros de la red, la ACH debió enfocar sus recursos de gestión hacia la activación y la movilización.

\section{Metodología y resultados}

Para el estudio del proceso de implementación de la Línea 4 se utilizaron dos tipos de fuentes de información. La primera consistió en la aplicación de entrevistas semiestructuradas a seis actores centrales vinculados con el proyecto. Entre ellos se encontraban funcionarios públicos de alto nivel de distintas dependencias del Gobierno del Distrito Federal y representantes de organismos civiles expertos en el tema de movilidad. Llegamos a estos actores a través de un muestreo de bola de nieve que inició con funcionarios de la $\mathrm{ACH}$. En este muestreo también se mencionaron agentes privados relacionados con el transporte público y con otras actividades económicas que se llevan a cabo en el Centro Histórico (transportistas, comerciantes y prestadores de servicios). Lamentablemente, varios actores no pudieron ser localizados o se negaron a dar información.

No obstante, y gracias a que el proceso de la Línea 4 estuvo ampliamente documentado, se pudo obtener información sobre el resto de los participantes a través del análisis de notas periodísticas, documentos oficiales y material publicado en las páginas electrónicas de los organismos 
involucrados en la discusión. La información proveniente de estas fuentes fue corroborada con la de las entrevistas para comprobar su validez y obtener una imagen lo más completa posible de la red de gobernanza del Centro Histórico y de su actuación en la implementación de la Línea 4. Toda la información recopilada se sometió a un análisis cualitativo de contenido. Con base en este método fue posible examinar los hechos y relaciones en el proceso estudiado, así como reconocer su significado en términos de los conceptos propuestos en el marco teórico sobre la gestión de redes colaborativas (Bryman, 2008).

\subsection{Caracteristicas de la red y su entorno}

La primera parte del análisis consistió en examinar las características de la red de gobernanza del Centro Histórico, respecto a los elementos seńalados por Provan y Kenis (2008). De acuerdo con los autores y con las proposiciones planteadas, se pueden señalar varios hallazgos. El primero es el grado de confianza entre los participantes. ${ }^{7}$ Como corresponde con el modelo, éste mostró ser bajo y -debido a la resolución que el GDF había tomado previamente respecto a la Línea 4- tenía que estar enfocado en los actores centrales, cuyo apoyo era vital para el proyecto. Aunque todos los participantes en la red son importantes por su capacidad de apoyar u oponerse a las decisiones tomadas por el GDF, había dos agentes clave. Por una parte, estaba el propio GDF y las diversas dependencias públicas relacionadas con el proyecto, además de la $\mathrm{ACH}$. Por otro lado se encontraban los transportistas, cuyas rutas y formas de organización se verían afectadas por la introducción del Metrobús.

Luego de que el GDF tomara la decisión de impulsar una cuarta línea de Metrobús, era esencial contar con el apoyo de los transportistas que trabajaban en la zona en cuestión. Tal apoyo no fue difícil de lograr, porque los concesionarios del transporte público conocían el éxito con que operaban las tres líneas construidas anteriormente. Los funcionarios entrevistados señalaron que, una vez que se logró convencerlos de iniciar

\footnotetext{
${ }^{7}$ Debe aclararse que, dada la naturaleza cualitativa de la investigación, no hay un parámetro de referencia claro para medir el grado de confianza entre los participantes. La deducción de que el nivel de confianza es bajo proviene del sentido de las reacciones y cuestionamientos que mostraron buena parte de los actores de la red de gobernanza del Centro Histórico. Dicho de otra manera, si se hubiera observado una aceptación generalizada de la propuesta del GDF desde el principio, se habría presumido una mayor confianza entre los miembros de la red. Algo similar ocurre con el número de participantes y con el consenso acerca de los objetivos de la red. Tampoco para estos elementos existe un parámetro objetivo de referencia. Sin embargo, de acuerdo con la evidencia que se ofrece sobre el caso, se puede argumentar que el número de participantes es moderado y que el consenso sobre los objetivos de la red es alto, como se menciona más adelante.
} 
el primer corredor por la Avenida de los Insurgentes, ${ }^{8}$ la rentabilidad económica del sistema y la mejora en las condiciones laborales de los operadores facilitó las negociaciones en la construcción de las siguientes líneas. A la Setravi correspondió el trabajo de gestionar, informar y persuadir a los concesionarios de que el Metrobús era una opción conveniente para beneficiar a los principales interesados: al GDF (para mejorar el servicio), a los propios empresarios (para generar un negocio rentable) y a los operadores de transporte (para mejorar sus condiciones de trabajo).

Pese a la importancia de su posición, debido a la naturaleza del proyecto, la relativa confianza de los concesionarios no fue suficiente para el éxito en la gestión de la red. Más allá de este hecho, otros miembros permanentes de la red (vecinos y comerciantes) mostraron el escepticismo que comúnmente presentan, cada vez que el GDF decide llevar a cabo obras de magnitud similar a la de la Línea 4 en el Centro Histórico. Como expresó uno de los entrevistados, "[nuestra] confianza no está ganada de antemano". De hecho, fueron estos actores quienes pusieron mayor resistencia al proyecto del Metrobús. Al respecto, uno de los funcionarios comentó que "el objetivo era que la mesa [de coordinación instalada por la $\mathrm{ACH}]$ [...] se convirtiera en una gran facilitadora para poder desarrollar el proyecto, [y para lograr] que se venciera la resistencia [de la comunidad]", que era muy fuerte. Otro de los involucrados ejemplificó la falta de confianza y de cohesión entre algunos de los actores del Centro Histórico: "No todos los comerciantes [...] son organizados, no todos se llevan entre ellos, no todos se hablan igual, no todos te atacan igual o te apoyan igual, no todos entienden de lo que les estás hablando, no todos están dispuestos al cambio".

En segundo lugar, destaca el número de participantes. Coincidiendo con lo que indica la tabla 1 , el número de integrantes de la red de gobernanza del Centro Histórico es moderado y manejable para la organización que la gestiona. Esto se debe a que, a pesar de haber algunos actores que destacan de manera individual, en su mayoría los vecinos y comerciantes se encuentran organizados y representados por pocas agrupaciones, y es a través de sus representantes que pueden involucrarse en la red. Además de esta característica global, hay que notar que el proyecto tuvo lugar en un corredor específico de la ciudad, y que quienes se manifestaron a favor y en contra de él eran principalmente las personas que vivían, trabajaban y tenían sus negocios cerca del área afectada por la nueva línea.

${ }^{8}$ La rentabilidad económica del BRT depende, en parte, de que atienda corredores de alta densidad poblacional. Como es de suponerse, al comenzar con vías con esta característica, como la Avenida de los Insurgentes, disminuye la probabilidad de que los concesionarios del transporte se opongan a futuros proyectos de este tipo. 
La tercera característica de la red es el consenso acerca de sus objetivos. Como se indicó, la ACH enfrentaba el reto de balancear el impulso a la actividad económica (comercio y servicios) y la recuperación del atractivo inmobiliario. Ambos objetivos (conservación y dinamismo económico) pueden ser considerados divergentes en naturaleza y están claramente manifestados en los documentos oficiales que rigen la actuación del GDF en esta zona del Distrito Federal, y en el mandato que se otorgó a la ACH. A ello contribuye la normatividad nacional e internacional a que está sujeta el área, debido a su calidad de patrimonio histórico. Por ejemplo, el primer objetivo que estableció el Plan Integral de Manejo del Centro Histórico, publicado en agosto de 2011, en la Gaceta Oficial del Distrito Federal fue "propiciar la recuperación del equilibrio urbano, social y económico del Centro Histórico asegurando la permanencia de sus valores culturales y la eficiencia del sistema urbano".

Contrario al modelo de Provan y Kenis (2008), el consenso al interior de la red del Centro Histórico respecto a estos objetivos no era moderadamente bajo sino alto, pues todos los integrantes los identifican, tácita o explícitamente. Además, parecen actuar conforme a ellos, sin que sea necesario discutirlos cada vez que se propone alguna medida concerniente al centro de la ciudad. Más aún, dado que los intereses de los agentes públicos y privados de la red están alineados con dichos objetivos, generalmente sirven de orientación para discutir los proyectos que el GDF propone para este punto de la capital.

Sin embargo, y aunque los objetivos son claros para todos los involucrados, es inevitable que algunos actores estén más interesados en promover, algunos de ellos en particular; por ejemplo, los empresarios y comerciantes se preocupan más por aquello que impacta el crecimiento de la actividad económica, mientras que las autoridades culturales independientes del GDF se muestran más interesadas por la conservación del entorno. En este contexto, es comprensible que el GDF y la $\mathrm{ACH}$, en particular, se posicione como la organización responsable de liderar la gestión de la red de gobernanza del Centro Histórico, puesto que tiene que defender y equilibrar ambos propósitos. Sobre este tipo de conflictos, un funcionario apuntó: "cualquier cambio [en el] sistema de movilidad [afecta] al comercio establecido en estas zonas [...] algunas zonas de vecinos presentan reticencias [...] Obviamente este tipo de acciones de gobierno se partidizan, $[y]$ entran intereses particulares o intereses políticos que van creando trabas al proyecto".

El cuarto componente se refiere a las competencias o habilidades a nivel de red. También en este aspecto existe diferencia con el modelo presentado en la tabla 1, puesto que la necesidad de competencias de la red que se identificaron son entre moderadas y altas. Esta característica 
se manifiesta, en primer lugar, en la especialización de las dependencias del GDF para atender las demandas particulares que surgieron, y para llevar a cabo las maniobras de negociación con líderes políticos y sociales, las cuales se volvieron necesarias y se describen en los apartados posteriores: la experiencia y habilidad de las dependencias en tratar movimientos de este tipo favorecieron también la resolución de los conflictos que afloraron en este caso. Al respecto, uno de los entrevistados señaló: "cada una de las entidades [del GDF] va aportando [lo que le corresponde] y es como, finalmente, se echa a volar ya este proyecto".

En segundo lugar, por la naturaleza del proyecto y los objetivos que sostiene la red, la información técnica era particularmente valiosa para defender el argumento de que el Metrobús era la mejor opción para la movilidad del Centro Histórico, así como para hacer adaptaciones razonables al proyecto en virtud de las demandas de los inconformes. Sobre este punto, uno de los actores involucrados en el proceso de gestión explicó:

\begin{abstract}
En [... la zona del CH de [las calles] Jesús María [y] Miguel Alemán el problema es con el ambulantaje, [...] y hubo que negociar también directamente con ellos para darles alternativas [...] En [la calle] Ayuntamiento los comerciantes establecidos, [...] veían difícil el suministro de sus insumos en los negocios, a ellos les hicimos [...] bahías de carga y descarga para que no interfirieran el ritmo del Metrobús. [...] Tampoco fuimos invasivos con las estaciones: en la zona, el perímetro del Centro Histórico, no hay estaciones sino estelas, nada más, que marcan el punto de ascenso y descenso de pasaje.
\end{abstract}

Además de las anteriores, se identificaron otras cualidades de la red de gobernanza del Centro Histórico que son importantes en su análisis. La primera es que algunos actores se conocían mutuamente desde tiempo atrás, debido a que habían participado en la discusión de otras medidas referentes al Centro Histórico, incluso antes de que la $\mathrm{ACH}$ fuera creada. Hay que considerar que parte de los organismos coordinados por la $\mathrm{ACH}$ son otras dependencias del GDF, como la Setravi y la SSP. Todas ellas, por pertenecer al mismo gobierno, están en contacto continuamente y dependen del Jefe de Gobierno.

En segundo lugar, está el hecho de que los transportistas ya habían enfrentado tres procesos de conversión al sistema Metrobús, muy similares al de la Línea 4, en lo que se refiere a los términos de las negociaciones con el gobierno. Si bien los transportistas no parecen pertenecer permanentemente a la red del Centro Histórico, ni mantienen siempre un interés preponderante en las decisiones sobre el centro de la ciudad como los comerciantes y los vecinos, su experiencia en proyectos semejantes fue aprovechada por la $\mathrm{ACH}$ a favor del proyecto de la Línea 4. 
Con el resto de actores involucrados en la red la relación es un tanto distinta porque, como los entrevistados comentaron, cada nueva acción requiere reuniones, negociaciones y acuerdos específicos.

Respecto a los recursos económicos para la ejecución del proyecto, la totalidad de ellos tenía que provenir de los actores clave mencionados antes, es decir, del GDF (que, a su vez, podía gestionarlos ante otras instancias, como el Gobierno Federal) y de los empresarios del transporte, quienes, en lugar de seguir invirtiendo bajo el modelo de concesiones hombre-camión, ahora lo harían mediante la empresa Metrobús. De esta forma, los recursos eran locales y estaban concentrados en los actores principales interesados en el proyecto.

Finalmente, también conviene tomar en cuenta que fue el GDF el que, luego de examinar técnicamente las posibles alternativas, concluyó que una línea de Metrobús era la opción más viable para la modernización del transporte en el área en cuestión. Así, el surgimiento del proyecto de la Línea 4 no se dio desde la interacción de la red de gobernanza del Centro Histórico, sino que fue una decisión tomada por el Gobierno de la Ciudad, que después se dio a conocer al resto de los actores. Hay que recordar que una de las características de las organizaciones líderes es que tienden a ajustar el comportamiento de la red a sus propios intereses.

\subsection{La actuación de la ACH en el proceso de gestión de la Línea 4}

El análisis de las características de la red del Centro Histórico no permite rechazar la proposición de que se trata de una red gestionada por una organización líder, con la salvedad de que algunos de sus atributos no corresponden con los del modelo conceptual. En particular, los objetivos y las competencias de la red estudiada no se ajustan con los que caracterizan a una red de ese tipo. Por sí sola, esta situación cuestiona la potencial efectividad del arreglo de gobernanza del Centro Histórico. Sin embargo, la evidencia recolectada sugiere que la red ha sido efectiva para alcanzar sus objetivos, no sólo porque logró la implementación de la Línea 4, sino también porque, de acuerdo con los entrevistados, ha contribuido exitosamente en la realización de otras obras y proyectos en el Centro Histórico, por ejemplo: la rehabilitación de espacios públicos, la peatonalización de algunas calles y la revitalización de algunas áreas, entre otras.

Con este antecedente, el análisis continúa indagando la manera en que una red intermediada por una organización líder, que se desvía del modelo conceptual, como la que tratamos, consiguió ser efectiva. En términos generales, es de esperarse que la respuesta está en el trabajo de gestión, es decir, en las acciones que llevan a cabo los gerentes de la red. Ante un escenario como el descrito, lo gerentes tendrían que dirigir los 
esfuerzos de la red y orientarla al cumplimiento de sus objetivos. Para explorar esta posibilidad, nos concentraremos en las proposiciones derivadas de McGuire (2002). En el resto de esta sección exploramos las acciones realizadas por la $\mathrm{ACH}$, distinguiendo la forma en que se gestionó a) el encuadramiento y la síntesis, b) la movilización, y c) la activación de la red durante el proceso de implementación de la Línea 4.

\section{a) Encuadramiento y sintesis}

Con el propósito de hacer avanzar la construcción de la Línea 4, la ACH estableció una mesa de coordinación y negociación, además de otros canales para el diálogo con los diversos actores de la red de gobernanza del Centro Histórico. Los entrevistados coinciden que fue mediante estos mecanismos que la $\mathrm{ACH}$ logró realizar las maniobras de encuadramiento y síntesis entre los diversos actores de la red. La mesa de coordinación cumplió con varias funciones: 1 . ofrecer información a los grupos que habían reaccionado negativamente al proyecto; 2 . proveer un espacio de diálogo con los inconformes para conocer las causas de su desacuerdo y proponer medidas para solucionarlas; 3. reunir a las dependencias del GDF relacionadas con el proyecto (y que también forman parte de la red del Centro Histórico), de modo que todas estuvieran involucradas en el proceso de negociación con los distintos actores, y participaran conjuntamente en la propuesta de alternativas a sus inquietudes y quejas; y 4. coordinar y dar seguimiento a todo el proceso de gestión y construcción de la nueva línea del Metrobús. De esta manera, la mesa buscaba convertirse en un foro cerrado de discusión entre los grupos que se sentían afectados por la propuesta de ampliar el Metrobús por el Centro Histórico.

El objetivo de "cerrar" la mesa era favorecer la confianza de los participantes para que expresaran de manera libre sus opiniones y, al mismo tiempo, hacer la discusión más manejable. En primer lugar, la ACH comunicó a los participantes que la decisión de reordenar el transporte público a través del Metrobús estaba ya tomada y no podía ser sujeta a discusión, toda vez que no rivalizaba con el objetivo de conservación del Centro Histórico y contribuía al de dinamismo económico, sobre todo desde el ángulo del turismo. También les informó a los participantes sobre los detalles del proyecto, el trazo de la ruta y las mejoras que se harían al entorno por donde pasaría el corredor.

De igual forma, la mesa de coordinación sirvió para que los diferentes grupos y personas interesadas manifestaran sus inquietudes sobre el proyecto, declararan las molestias que a su juicio ocasionaría la obra -tanto durante su construcción como en la operación del Metrobús-, y para que, entre todos los participantes de la mesa, encontraran una solución a cada 
demanda individual, si se consideraba legítima. Una de las intenciones de la $\mathrm{ACH}$ era que la información fluyera entre los actores a través de la mesa para que tuvieran una imagen clara y directa de las implicaciones colectivas e individuales del proyecto, y para que las soluciones a los problemas planteados por los vecinos, comerciantes, empresarios y demás grupos fueran acordadas conjuntamente, asegurando su apoyo o, cuando menos, su no oposición a la Línea 4.

Fue en el marco de la interacción propiciada en la mesa en el que pudo definirse con mayor exactitud dónde era necesario que intervinieran las dependencias del GDF, como la Secretaría de Gobierno, la Setravi, la SOPS y la SSP, en virtud de su área de especialidad. Así, la información y el intercambio resultantes de la mesa de coordinación contribuyeron a las acciones de movilización y activación. Por ejemplo, la Secretaría de Gobierno se percató de quiénes eran los agentes que no aceptarían los arreglos de la mesa, y que probablemente contaban con apoyo y financiamiento de actores políticos pertenecientes a partidos opositores al GDF para seguirse manifestando en contra del proyecto. Además, la SOPS pudo evaluar si los cambios en la ruta o las obras complementarias que se pedían eran viables. Finalmente, la SSP dejó claro a los comerciantes ambulantes que no se permitiría que siguieran trabajando a lo largo del corredor, y que tomaría medidas coercitivas al respecto en caso de ser necesario.

\section{b) Movilización}

Las actividades de movilización se centraron en dos grupos de actores. El primero de ellos fue el de los concesionarios del transporte público que explotaban las rutas desplazadas por el Metrobús, y que era uno de los actores clave para la puesta en marcha del proyecto de la Línea 4. En la división del trabajo de la red coordinada por la $\mathrm{ACH}$, el trato con este grupo correspondió a la Setravi. Desde el establecimiento de la primera línea del Metrobús, el grupo más importante con el que hubo que llegar a acuerdos fue el de los concesionarios del transporte público. Esto debido a que la adopción del Metrobús exige cambios en la forma de organizar y administrar el negocio del transporte.

De acuerdo con la Ley de Transporte y Vialidad del Distrito Federal, el GDF puede otorgar concesiones a particulares para la prestación del servicio, sin que puedan asignarse más de cinco a una misma persona física o moral. Esta forma de trabajo recibe comúnmente el nombre de "sistema hombre-camión". Aunque todas las rutas de autobuses y microbuses operan con base en este mecanismo, el sistema Metrobús se organizó de forma diferente con el fin de prestar un mejor servicio a los usuarios. Desde la creación de la Línea 1, el Metrobús se constituyó como 
una empresa donde los principales socios son los transportistas que anteriormente operaban mediante concesiones. De esta forma, los operadores no tendrían que competir por los pasajeros para tener mayores ingresos lo que, junto con la tecnología que el sistema trae consigo y el hecho de transitar sobre un carril exclusivo, resultaría en una mejora sustancial en el transporte público.

Amparados en la experiencia positiva de las tres líneas anteriores, para la Setravi fue relativamente sencillo y rápido convencer a los concesionarios de que un corredor en el Centro Histórico también parecía convenir a la mayoría de los participantes (transportistas, usuarios, gobierno, etcétera) y que tenía grandes posibilidades de éxito. El proceso de negociación con los empresarios del transporte siguió un proceso similar a las negociaciones de líneas previas, en el que los particulares suelen encontrar ventajas: al devolver sus concesiones a la Setravi y sumarse a la empresa Metrobús, los antiguos concesionarios se vuelven accionistas y se convierten en sujetos de crédito para adquirir unidades de Bus Rapid Transit de alta tecnología. Los antiguos choferes de ruta, por su parte, también se incorporan a la empresa, la cual elige de entre ellos a quienes serán — después de la capacitación correspondiente- los operadores del Metrobús. Para los antiguos choferes de microbús, la principal ventaja de ser parte de la empresa es recibir prestaciones que no tenían con el anterior sistema de trabajo.

El segundo grupo de actores movilizado fueron los vecinos y comerciantes establecidos que se mostraban escépticos sobre la forma en que la construcción y el funcionamiento del corredor les afectarían. El principal liderazgo en este conjunto fue el del presidente del Consejo de Asociaciones de Comerciantes y Vecinos del Centro Histórico (CACV). A esta organización se le informó sobre los beneficios económicos que traería la Línea 4: más gente, mayores ventas y más empleo. El CACV estuvo dispuesto a negociar y a abordar el tema del Metrobús, al mismo tiempo que ofreció un apoyo moderado al proyecto. Las razones de su renuencia al funcionamiento de la Línea 4 eran básicamente tres: que si las obras se prolongaban demasiado afectarían las ventas; que si el GDF no ofrecía alternativas o apoyos a los comerciantes pequeńos, estos se verían en riesgo de cerrar definitivamente sus negocios, incluso más allá del periodo de construcción; y finalmente, que el paso del Metrobús estorbaría el acceso para las maniobras de carga y descarga que tienen que hacer los proveedores y los clientes, ocasionando afectaciones en costos y ventas.

Sobre los dos primeros puntos no hubo mayor dificultad porque las autoridades ofrecieron, a través de la $\mathrm{ACH}$, que la obra se concluiría en un plazo breve y razonable, lo que ocurrió de la forma prevista. Por otra parte, el GDF no otorgó los apoyos solicitados durante la construcción, 
por considerarlo innecesario. Con respecto a la tercera causa de oposición al proyecto, la $\mathrm{ACH}$ llevó a discusiones detalladas dentro de la mesa de coordinación. En ella estuvieron presentes los propietarios, vecinos y comerciantes interesados, con quienes se negoció individualmente. Las autoridades realizaron acuerdos particulares, cuadra por cuadra del recorrido del Metrobús, otorgando permisos para que los clientes o los proveedores pudieran ocupar momentáneamente la calle y realizar los movimientos de mercancía. En otros casos, se ofreció, además, construir bahías de carga y descarga en calles aledañas a los negocios donde fuera posible su instalación. Zanjadas estas inquietudes, los vecinos y comerciantes no pusieron mayor reparo a la Línea 4.

\section{c) Activación}

Este proceso se llevó a cabo desde un principio con los empresarios del transporte público, en su rol de actores clave en el proyecto. Sin embargo, hubo también agentes interesados en influir en la propuesta del Metrobús del Centro Histórico, poniendo en juego sus conocimientos, experiencias, relaciones y demás recursos para impedir que se llevara a cabo. En este aspecto, la labor de la autoridad y de las dependencias coordinadas del GDF estuvo orientada a "desactivar" esos movimientos. Las manifestaciones de los comerciantes establecidos en el Centro Histórico fueron las más documentadas por la prensa, en lo que se refiere a los conflictos generados por la construcción de la Línea 4. Algunas de esas manifestaciones, por ejemplo, se vincularon con presiones políticas provenientes de los partidos de oposición en el Distrito Federal, según el propio GDF.

En la oposición al proyecto se distinguieron dos liderazgos entre los comerciantes y vecinos del Centro Histórico: la Unión de Comerciantes Establecidos de La Merced (UCEM), y la Unión de Vecinos y Comerciantes de la Poligonal del Centro Histórico (UVCP). Las organizaciones utilizaron diferentes estrategias y argumentos. La UCEM advirtió que la ciudad (y concretamente la zona de La Merced) perdería su atractivo popular y el dinamismo comercial que la caracteriza. La UVCP fue más aguerrida, a decir de las autoridades del Distrito Federal. Al igual que la UCEM, la UVCP organizó manifestaciones de vecinos y comerciantes, aunque fue más allá al interrumpir, en algunas ocasiones, la construcción de la obra.

La UVCP argumentaba que el proyecto no estaba técnicamente bien sustentado. Sobre este punto lograron el apoyo de los profesores-investigadores de la delegación sindical D-II-IA-1 del INAH quienes, el 5 de julio de 2011, publicaron un desplegado en periódicos nacionales, como Excélsior. En este desplegado manifestaban su respaldo a la UVCP y des- 
calificaban el dictamen aprobatorio que la Coordinación Nacional de Monumentos Históricos del propio INAH había otorgado al proyecto del Metrobús, ${ }^{9}$ alegando el incumplimiento con una serie de estudios técnicos y sociales. Entre las demandas más importantes, postuladas por la UVCP hacia la ACH y el GDF, estuvieron la realización de una consulta ciudadana para decidir si se establecía o no la Línea 4, y la petición de que se hicieran modificaciones en el recorrido proyectado. El GDF respondió que no se cambiaría el trazo de la ruta del Metrobús y que no habría tal consulta, aunque mantendrían el diálogo con los comerciantes $\mathrm{y}$ vecinos.

Con el fin de restarle legitimidad a las demandas de la UVCP y su lideresa, el GDF y la ACH señalaron que esta organización estaba vinculada con el Partido Revolucionario Institucional (PRI). Con esto cuestionaban la legitimidad del movimiento y afirmaban que éste no obedecía a una preocupación genuina por el Centro Histórico, sino al proceso electoral que estaba próximo, donde se elegiría al nuevo jefe de gobierno y a los jefes delegacionales. La Secretaría de Gobierno del Distrito Federal fue la encargada de desarticular las protestas de la UVCP, negociando directamente con los líderes políticos que las encabezaban y financiaban.

La figura 1 resume el proceso y las cuatro actividades centrales de gestión coordinadas por la $\mathrm{ACH}$ durante la implementación de la Línea $4 .{ }^{10}$ Como puede apreciarse en el esquema, no se trató de un procedimiento lineal o por etapas, sino que fue un trabajo simultáneo en cada uno de los frentes, que permitió afianzar la aceptación del proyecto e implementarlo exitosamente.

9 También el Centro del Patrimonio Mundial de la UNESCO había dado su aval al proyecto. Ambas instituciones negaron que fueran a provocarse dańos a los edificios o a la infraestructura del Centro Histórico.

${ }^{10}$ Ramírez (2016) sostiene que hay tres formas de conceptualizar a las redes: como estructuras, como relaciones y como instrumentos. Esta investigación utilizó un enfoque de redes como relaciones, debido a que esta perspectiva permite explicar la manera en que la organización de la red y sus reglas intervienen en la definición y solución de los problemas públicos. A diferencia de un enfoque estructural, la visión relacional de redes no tiene como propósito lograr una representación gráfica de la red estudiada, en términos de nodos y lazos. 


\section{Figura 1}

\section{La actuación de la ACH en el proceso de gestión de la Línea 4 del Metrobús}

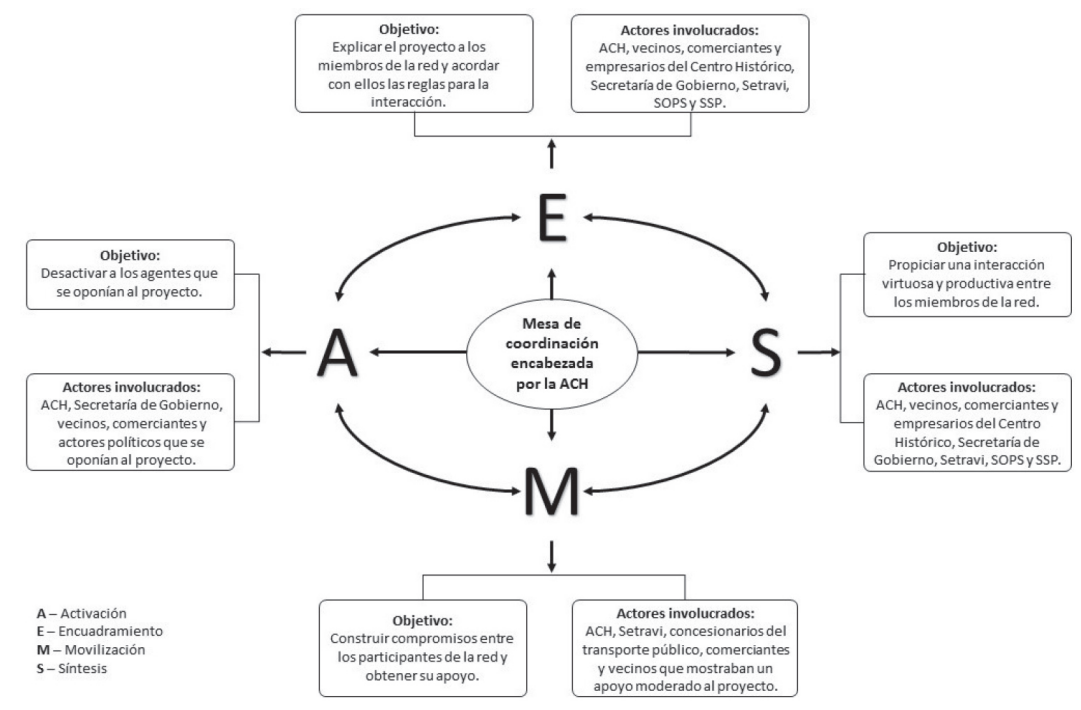

Fuente: elaboración propia.

\section{Discusión}

Dos aspectos sobresalen de los hallazgos presentados en la sección anterior. Por una parte, es posible identificar que la red tenía objetivos relativamente bien definidos y claros para la mayoría de los participantes, lo que indica que fueron adecuadamente socializados entre los diversos actores. Por otro lado, es importante discutir cómo la distribución de los recursos (económicos, políticos, etcétera) entre los agentes afectan la gestión de la red.

Los hallazgos presentados en el apartado anterior no proveen suficiente evidencia para rechazar las proposiciones 2 y 3 . Es decir que, dada la claridad de objetivos de la red, en efecto ésta enfocó sus esfuerzos hacia la activación y la síntesis, y dedicó menos recursos a la movilización y el encuadramiento de sus integrantes. Sin embargo, son necesarias algunas precisiones. En el marco de una clara definición de los objetivos, la organización líder se ocupó de una importante labor de síntesis, mediante el establecimiento de una mesa de coordinación y negociación. No obstante, a pesar de la claridad de los objetivos, la misma mesa tuvo que servir para tareas de encuadramiento, necesarias, no sólo porque la $\mathrm{ACH}$ es un órgano de reciente creación, que tiene que consolidar su posición como líder, sino también porque cada nuevo proyecto diseñado para el Centro 
Histórico requiere discutirse desde el origen con quienes participan en la gobernanza de esa zona de la ciudad.

Esas discusiones, que se llevan a cabo en arenas específicas de política pública, se definen en función de la forma en que el proyecto afecta, tanto la promoción económica como la preservación del Centro Histórico. Un proceso similar a éste permitió a la $\mathrm{ACH}$, en el caso de la Línea 4, alcanzar acuerdos y distribuir las tareas necesarias para el logro de la implementación del corredor, e incluso para la coaptación de algunos actores. Es destacable que el encuadramiento no estuvo dirigido hacia los objetivos generales del arreglo, sino a lograr que los participantes aceptaran que el proyecto de la Línea 4 era un instrumento viable para mejorar la movilidad del Centro Histórico y que contribuía a cumplir los objetivos acordados con los miembros de la red.

Esto nos lleva a subrayar la importancia de diferenciar entre los objetivos formales (globales) de la red, y los objetivos intermedios o instrumentales (la Línea 4). La evidencia empírica indica que, al existir un alto consenso en torno a los objetivos al interior de una red liderada, aún puede presentarse un desplazamiento hacia la discusión de los instrumentos que deben utilizarse. Esto sugiere dos posibilidades: 1. que la conformidad con los objetivos globales no lleva implícita la aceptación de cualquier herramienta u objetivo intermedio para alcanzarlos, independientemente de la pertinencia teórica o técnica que tenga para algunos actores; y que, por lo tanto, 2. el consenso sobre los medios o instrumentos que la red podría emplear para sus propósitos puede ser tan importante como cualquiera de los otros elementos propuestos por Provan y Kenis (2008).

Los resultados tienen, además, implicaciones opuestas a lo que sostiene la proposición 4, pues indican que, aunque algunos o todos los participantes de la red hayan trabajado juntos antes, eso no garantiza que deban dirigirse pocos recursos de gestión a las acciones de síntesis. Además, los resultados aportan elementos relevantes para comprender las implicaciones empíricas de la proposición 5, la cual estableció que, si la red en cuestión tenía objetivos relativamente claros, la $\mathrm{ACH}$ debió enfocar gran parte de sus recursos de gestión a explotar el ambiente externo a favor de la Línea 4, más que a estabilizar el sistema al interior de la red. Considerando que la $\mathrm{ACH}$ tenía que llevar a la red hacia el establecimiento de la Línea 4 del Metrobús en un entorno de escepticismo y de resistencia parcial al cambio, sí se observó -contrario a la expectativa hipotética- un gran esfuerzo por estabilizar el ambiente al interior de la red. La necesidad de dedicar esfuerzos a estabilizar la red se explica, en parte, por el hecho de que de ello dependía que se consolidara el propósito del gobierno, y que se asegurara la construcción y la operación correcta del Metrobús. 
En resumen, la evidencia sugiere que la claridad de objetivos y el acuerdo en ellos no aseguran que la activación y la síntesis sean suficientes para una adecuada gestión de la red. Por otra parte, tampoco es posible afirmar que la ausencia o inexactitud de dichos objetivos hagan necesario que todo el esfuerzo del gestor de la red se dedique al encuadramiento. De la misma forma, no se puede afirmar que el consenso sobre los objetivos globales de la red sea suficiente para asegurar la estabilidad al interior de la misma. Finalmente, los resultados indican que la claridad y el consenso sobre los objetivos globales sí proporcionan un piso más estable para la negociación de los instrumentos u objetivos intermedios que se usarán en el alcance de los propósitos generales de la red.

Con respecto a los recursos (financieros y de otros tipos) los resultados sugieren que no se encontraban dispersos, sino concentrados en el propio GDF y los concesionarios del transporte público afectados. Estos mismos agentes apoyaron el proyecto prácticamente desde el principio, gracias al éxito observado en las experiencias de los corredores que ya funcionaban, y por los beneficios económicos y laborales que los transportistas podrían obtener. En este sentido, no podemos rechazar la proposición 6, que sugería que, de haber apoyo al proyecto, la $\mathrm{ACH}$ debía invertir menos gestión en movilizar a los diversos actores. Como se describió antes, la movilización de los empresarios del transporte fue, en efecto, una de las partes relativamente más sencillas y rápidas de todo el proceso, y requirió invertir comparativamente menos recursos de gestión.

En el mismo sentido, tampoco podemos rechazar la proposición 7 que sugiere que, si los recursos están poco dispersos se dirigen menos esfuerzos de gestión hacia la movilización. Sin embargo, la relación con la activación no parece clara en nuestro caso de estudio, toda vez que se dio en un sentido negativo, pues tuvo como meta identificar a los actores que poseían recursos para oponerse al proyecto para neutralizar su intervención. Contrario a lo que puede interpretarse de la proposición 7 , no hay una relación clara entre recursos no dispersos y maniobras de (des)activación. El enunciado podría replantearse para señalar que una menor dispersión de recursos se corresponde con menores esfuerzos de activación, salvo en el caso que haya agentes cuyo propósito sea contrario al programa que impulsa la red. Finalmente, aunque tampoco podemos rechazar la proposición 8 , que sostiene que si los recursos son locales la organización líder dirigirá sus acciones hacia la movilización y la activación, puede sugerirse que el concepto de activación también considere los aspectos negativos, como los que ya se señalaron.

Si en la primera parte de la discusión se habló del desplazamiento del debate hacia los instrumentos que podría usar la red para alcanzar sus objetivos, ahora debe resaltarse que la realización de las actividades de 
gestión involucradas en las proposiciones requería competencias y habilidades especializadas a nivel de red. Estas competencias fueron puestas en práctica por las diversas dependencias del GDF, sobre todo - aunque no exclusivamente- en las actividades de encuadramiento y activación, y sin ellas los resultados positivos no hubieran sido posibles. La ausencia de tales habilidades habría puesto en riesgo la efectividad de la red, dada la importancia que adquirió el encuadramiento para alinearla hacia la aceptación de la Línea 4, y por la relevancia que tuvo la desactivación de los movimientos contrarios al proyecto. En suma, ambas actividades resultaron ser clave para el éxito de la implementación del corredor.

\section{Conclusiones}

Los resultados de la investigación apoyan el argumento de que los mecanismos de gestión en red pueden contribuir de manera importante a tratar con los problemas que se presentan en escenarios fragmentados, donde participan grupos y actores con intereses altamente diversos. Por esta misma razón, se confirma que la gestión colaborativa por medio de redes puede ayudar también a incrementar la efectividad de las acciones públicas. Este tipo de gestión se requiere en las ciudades mexicanas, caracterizadas por contextos poco democráticos, altamente jerarquizados y espacios políticos en disputa. La gestión por medio de redes puede contribuir, en la práctica, a la inclusión de diversos grupos, lo mismo que a proporcionar mayor flexibilidad en las negociaciones, y a neutralizar o coaptar a ciertos agentes.

Se encontró que una red con una estructura gestionada por una organización líder puede ser efectiva a pesar de que sus características no se apeguen a las expectativas del modelo teórico. La evidencia apunta a que, cuando esto ocurre, el esfuerzo de los gestores de la red se enfoca a enfrentar las consecuencias de estas desviaciones mediante el énfasis en ciertas acciones de gestión. Específicamente, observamos que, ante objetivos consensuados y el desplazamiento de la discusión hacia los instrumentos que puede utilizar la red para alcanzar sus propósitos, gran parte de los esfuerzos se dirigen hacia el encuadramiento y la activación (o "desactivación", según el caso). Esto lleva a reconsiderar algunos aspectos de las proposiciones de McGuire (2002) sobre los comportamientos de los gestores ante determinados escenarios. Un hallazgo interesante, por ejemplo, es que conviene considerar a la activación en dos sentidos, tanto en el positivo, de quienes contribuyen a que los propósitos se cumplan, como en el negativo, que abarca a quienes trabajan en sentido contrario.

Por otra parte, el caso analizado sugiere que el consenso sobre los medios, dado los objetivos globales que la red pretende alcanzar, puede ser un factor igual de importante que los otros cuatro predictores de la 
efectividad de la red, propuestos por Provan y Kenis (2008). Finalmente, las alteraciones señaladas en la conformación de la red, y su "compensación" a través de las labores de gestión, pueden llevar a una estructura como ésta a requerir mayores competencias y habilidades que las que se le asignarían de acuerdo con el modelo teórico original.

\section{Fuentes consultadas}

Agranoff, Robert (2003), Leveraging networks: a guide for public managers working across organizations, IBM Center for the Business of Government, Arlington.

Agranoff, Robert (1991), "Human Services Integration: Past and Present Challenges in Public Administration", Public Administration Review, 51 (6), American Society of Public Administration, Washington, pp. 533-542.

Berry, Frances S., Ralph S. Brower, Sang O. Choi, Wendy X. Goa, HeeSoun Jang, Myungjung Kwon y Jessica Word (2004), “Three traditions of network research: what the public management research agenda can learn from other research communities", Public Administration Review, 64 (5), American Society of Public Administration, Washington, pp. 539-552.

Bryman, Alan (2008), Social Research Methods, Oxford University Press, New York.

McGuire, Michael (2006), "Collaborative public management: assessing what we know and how we know it", Public Administration Review, 66 (Special Issue), American Society of Public Administration, Washington, pp. 33-43.

McGuire, Michael (2002), "Managing networks: propositions on what managers do and why they do it", Public Administration Review, 62 (5), American Society of Public Administration, Washington, pp. 599-609.

O’Toole, Laurence J. (1997), “Treating networks seriously: practical and research-based agendas in public administration", Public Administration Review, 57 (1), American Society of Public Administration, Washington, pp. 45-52. 
Provan, Keith G. y Patrick Kenis (2008), "Modes of network governance: structure, management, and effectiveness", Journal of Public Administration Research and Theory, 18 (2), Public Management Research Association, Washington, pp. 229-252.

Provan, Keith G. y Brinton Milward (1995), "A preliminary theory of interorganizational network effectiveness: a comparative study of four community mental health system", Administrative Science Quarterly, 40 (1), Cornell University, Ithaca, pp. 1-33.

Ramírez, Edgar E. (ed.) (2016), Análisis de redes sociales para el estudio de la gobernanza y las políticas públicas. Aproximaciones y casos, CIDE, México.

Ramírez, Edgar E. (2012), "Instituciones y gobernanza metropolitana: una primera aproximación al caso de México”, Estudios Demográficos y Urbanos, 27 (2), El Colegio de México, México, pp. 491-520.

Rhodes, Rod A. W. (2000), "Governance and public administration”, en Jon Pierre (ed.), Debating Governance. Authority, Steering and Democracy, Oxford University Press, Oxford, pp. 54-90.

Salancik, Gerald R. (1995), "WANTED: a good netwok theory of organization", Administrative Science Quarterly, 40 (2), Cornell University, Ithaca, pp. 345-349.

Wright, Lloyd, Walter Hook y Carlos F. Pardo (2010), Guia de planificación de sistemas BRT, Institute for Transportation \& Development Policy, New York.

Recibido: 22 de octubre de 2015. Corregido: 11 de abril de 2016. Aceptado: 8 de junio de 2016.

Edgar E. Ramírez-de la Cruz. Mexicano. Doctor en administración y políticas públicas por la Universidad Estatal de Florida. Actualmente es profesor-investigador del CIDE. Su trabajo ha sido publicado en revistas académicas como The American Journal of Political Science, International Review of Public Administration, International Journal of Public Administration, Urban Affairs Review y Public Administration Review, así como en diversos capítulos en libros editados en México, China y Estados Unidos. Su principal interés es el estudio de la gobernanza urbana. Sin embargo, sus líneas de investigación incluyen gestión del crecimiento, regulación 
del uso del suelo, redes de política pública y reforma de la administración pública. Publicaciones recientes, en coautoría: "Controlling administrative discretion promotes social equity? Evidence from a natural experiment", Public Administration Review, American Society of Public Administration, Washington, pp. 1-10 (2016); "Línea Verde, an Axis Road in Aguascalientes, Mexico", en William G. Holt (ed.), From sustainable to resilient cities: global concerns and urban efforts, Emerald Group Publishing Limited, Somerville, pp. 215-237 (2014); "Estrategias dilemas y oportunidades de la nueva misión y visión del Infonavit: un estudio de caso", Gestión y Política Pública, 23 (1), Centro de Investigación y Docencia Económicas, México, pp. 121-183 (2014).

Manlio F. Castillo-Salas. Mexicano. Doctor en políticas públicas por el CIDE e investigador por proyecto en la División de Administración Pública de la misma institución. Sus intereses y líneas de investigación se relacionan con políticas públicas, regímenes urbanos, gobernanza urbana, servicios públicos y desarrollo regional. 